\title{
Functional Tasks performed by People with Severe Muscular Atrophy using an sEMG controlled Robotic Manipulator
}

\author{
Annette Hagengruber and Jörn Vogel.
}

\begin{abstract}
For paralyzed people activities of daily living like eating or drinking are impossible without external assistance. Robotic assistance systems can give these people a part of their independence back. Especially if the operation with a joystick is not possible anymore due to a missing hand function, people need innovative interfaces to control assistive robots in 3D. Besides brain computer interfaces an approach based on surface electromyography (sEMG) can present an opportunity for people with a strong muscular atrophy. In this work we show that two people with proceeded spinal muscular atrophy can perform functional tasks using an SEMG controlled robotic manipulator. The interface provides a continuous control of three degrees of freedom of the endeffector of the robot. The performance was assessed with two clinical measures of upper limb functionality: the Box and Blocks Test and the Action Research Arm Test. Additionally, the participant could show that they can drink by themselves with the provided system.
\end{abstract}

\section{INTRODUCTION}

Stroke, trauma or neuromuscular diseases can lead to strong physical handicaps. In severe cases all four limbs are affected and no voluntary limb movements can be realized. For people affected, activities of daily living like eating and drinking become a huge challenge and full time care is inevitable. Assistive devices can help these people to partly get their independence back.

For interaction with the environment and to execute functional tasks, robotic devices can be used. Systems like the MANUS of iArm [1], or the JACO of Kinova [2], are some of the few commercially available devices. Both devices consist of a robotic arm with six degrees of freedom and a gripper to allow for manipulation of objects. These assistive robots are usually controlled by a joystick, which offers an easy, intuitive, and continuous interface. For people unable to handle a joystick, alternative interfaces have to be used. Here, Brain Computer Interfaces (BCI) present one viable option for completely paralyzed people. In BCI research, two classes of interfaces are distinguished, invasive and non-invasive ones. Both classes of interfaces have been used to achieve control over robotic system. In case of non-invasive methods like electroencephalography (EEG), the robot can be controlled using discrete control commands [3], or EEG-signals in combination with eye tracking [4]. Invasive methods, where signals are typically recorded directly from cortical areas of the brain, have proven to allow for continuous control of an assistive robot in 3D [5]-[7].

This work was supported by the Bavarian Ministry of Economic Affairs, Energy and Technology (StMWi)

The authors are with the Institute of Robotics and Mechatronics, German Aerospace Center (DLR), Oberpfaffenhofen/Germany

Contact: annette.hagengruber@dlr.de
An alternative approach to achieve continuous control signals, is the usage of surface electromyography (sEMG). sEMG is a well known method in the field of prosthetics, where recordings of muscular activity are used to control prosthetic hands and perform functional tasks of daily living [8], [9]. In [10] it could be shown that sEMG can be used to record residual muscle function from people with severe spinal muscular atrophy. Although the residual activity does not suffice to exert functional motion of the limbs, the recorded data could be used to control a 3D simulation of a robotic arm. Thus, such an interface could present a viable non-invasive approach for paralyzed people with residual muscle function to control an assistive robot and thus perform tasks of daily living.

In this work we show that two paralyzed people with severe muscular atrophy can perform reach and grasp tasks with a light-weight robot and an sEMG-based interface. The interface provides the user with continuous control of three degrees of freedom simultaneously. The performance of the control was assessed with two clinical measures of manual dexterity and upper limb function: the Box and Blocks Test and the Action Research Arm Test (ARAT). A simplified control strategy was used to perform the Box and Blocks Test, whereas the ARAT test was executed to assess the full control over seven degrees of freedom (translation, rotation and grasp).

\section{Methods}

The experiments presented in this work show the use of a light-weight robot with an sEMG-based interface used by people with paralysis in a functional tasks. The interface allowed the participants to continuously command the endeffector-velocity of the robot simultaneously in three degrees of freedom. The performance was evaluated using two clinical assessment tests: The Action Research Arm Test (ARAT) and the Box and Blocks Test (B\&B). Additionally the participants performed a drinking task with the provided system. In total all experiments were done on four consecutive days. The participants had no further training to these experimental days.

\section{A. Participants}

Two 49-year old women (S1 and S2), both suffering from spinal muscular atrophy (SMA) type II took part in the experiments. SMA leads to the death of the motor neurons in the spinal cord resulting in a progressive muscle weakness. In $\mathrm{S} 1$ and S2, the progressive disease has proceeded so far, that due to muscle atrophy, almost no voluntary limb movement 
TABLE I

LIST OF MUSCLES OF THE USED SENSOR CONFIGURATION

\begin{tabular}{cc} 
Electrode & Participant 1 \\
\cline { 2 - 2 } 1 & thenar \\
2 & hypothenar \\
3 & flexor carpi radialis \\
4 & extensor digiti minimi \\
5 & brachioradialis \\
6 & extensor carpi ulnaris \\
7 & triceps brachii \\
8 & pectoralis major
\end{tabular}
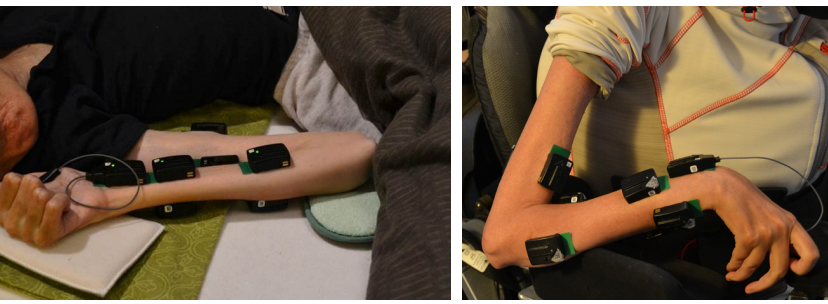

Fig. 1. Placement of the electrodes at the right arm of the participants; Eight surface EMG-sensors were used respectively. Left: shows the sensor placement of S1; Right: shows the sensor placement of S2.

To realize the interface, different combinations of muscle activity are recorded for different control signals. This means, each control signal is generated from a mixture of muscles signals, defined individually by the user. The recorded data is mapped to the control signals using machine learning methods based on Gaussian Processes. Here, a three dimensional velocity command $( \pm \mathrm{x}, \pm \mathrm{y}, \pm \mathrm{z})$ and a binary trigger signal $(0,1)$ are decoded. To acquire the mapping, a training procedure has to be performed before the usage. First, the muscular activity emerging when the user is not activating any muscle (resting) is measured as baseline. Afterwards, the user is sequentially asked to provide muscular activity which shall be associated with motion along directions of the cardinal axes (right, left, forward, backward, up and down). Each directional activity is recorded for 2 seconds followed by at least one second of resting. Due to the known sequence of directions, the recorded sEMG data can be labeled accordingly. Typically three repetitions of the training cycle are recorded to achieve variation in the muscular activity. From the sEMG data, four different features are calculated:

- sEMG-amplitude

- Slope-Sign-Change

- Zero-Crossing-Rate

- Waveform-Length

The digitization and processing of the sEMG signals happens at a rate of $1 \mathrm{kHz}$ on a linux real-time machine. Gaussian Process Regression is then used to decode the three dimensional desired velocity vector. Training data for the trigger signal is recorded by repeated tension and relaxation of the respective muscle-combination to be associated with the trigger signal. From this data the binary trigger signal is decoded using a classifier based on Linear Discriminant Analysis (LDA). Since sEMG decoding is very sensible to the positioning of the electrodes (especially in users with atrophic muscles) the training procedure was conducted in the beginning of every experimental session.

\section{Robot Control}

A DLR Light-Weight Robot III (LWR3) equipped with a five-fingered DLR-HIT HAND was used in these experiments. During the experiments the robotic arm was always operated in Cartesian impedance control [11]. This provides a stable behavior of the robot even in contact with the environment and allows for control of the robots endeffector-velocity based on the decoded sEMG commands. wrapped with medical cohesive tape Fig 1 shows exemplar electrode placement for both participants. 


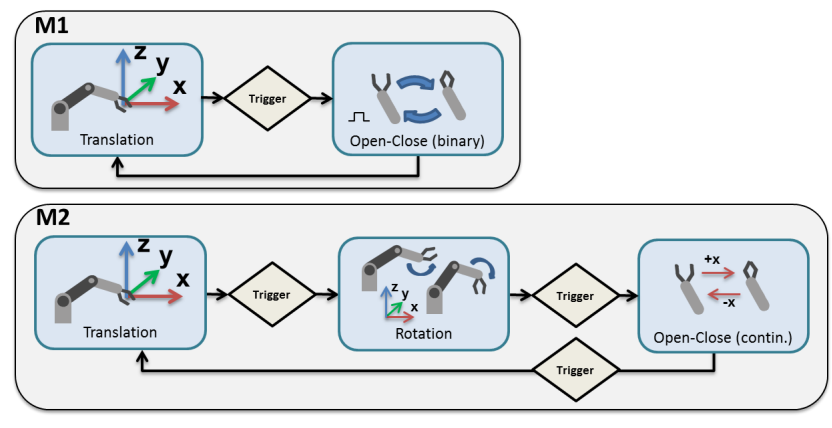

Fig. 2. Illustration of the modes M1 and M2; M1 includes only translational control with a binary grasp-trigger, whereas M2 allows to sequentially switch control between translation, rotation, and continuous opening and closing of the hand.

Furthermore, the dedicated torque sensing of the robot allows for detection of external forces. To protect the hardware, a safety mode is activated, whenever external forces pass a predefined threshold [12]. Similar to the robot, the fivefingered hand is equipped with joint torque sensors, which allow for joint impedance control in order to provide versatile and compliant grasping capabilities.

Two different modes were implemented to allow for mapping of the decoded velocity to endeffector velocity.

- Mode 1 (M1): This mode allows the user to control only the translation. Thus, the decoded velocity commands allow to move the endeffector continuously in three degrees of freedom $( \pm \mathrm{x}, \pm \mathrm{y}, \pm \mathrm{z})$. Combined commands are possible as well (moving diagonal). The velocity of the endeffector in space is determined by the strength of the muscle activity up to a maximum of $0.2 \mathrm{~m} / \mathrm{s}$. In M1 the orientation of the robotic hand cannot be changed by the user. The binary trigger signal is used to switch the finger configuration of the robotic hand between a predefined open and close posture. The method is illustrated in Fig. 2.

- Mode 2 (M2): Mode M2 provides the user with the control over all 7 degrees of freedom, i.e. the translation and rotation of the endeffector and the aperture of the hand. The submodes of translation, rotation, and finger-movement can sequentially be selected using the trigger signal. An overview of the control mode is illustrated in Fig. 2 at the bottom. The controlling of translations is equivalent to that described in $\mathrm{M} 1$. In rotational control, $\pm \mathrm{x}$ is mapped to a rotation around the $y$-axis, $\pm y$ is mapped to a rotation about the $\mathrm{z}$-axis, and $\pm \mathrm{z}$ is mapped around the $\mathrm{x}$-axis. In previous tests, this mapping emerged as most intuitive. To simplify rotational control, only the most dominant decoded velocity command was applied, i.e. no simultaneous rotation around multiple axes was possible. In M2 the closing and opening of the hand can be controlled continuously in the third submode by commanding $+x$ for closing and $-x$ for opening. To support the user, a monitor was used to display the currently active sub-mode. Additionally, an audio signal was played to indicate the change between sub-modes.

Due to safety reasons a maximum velocity of $0.2 \mathrm{~m} / \mathrm{sec}$ was allowed in these experiments although the participants were located outside the reach of the robot. By observing

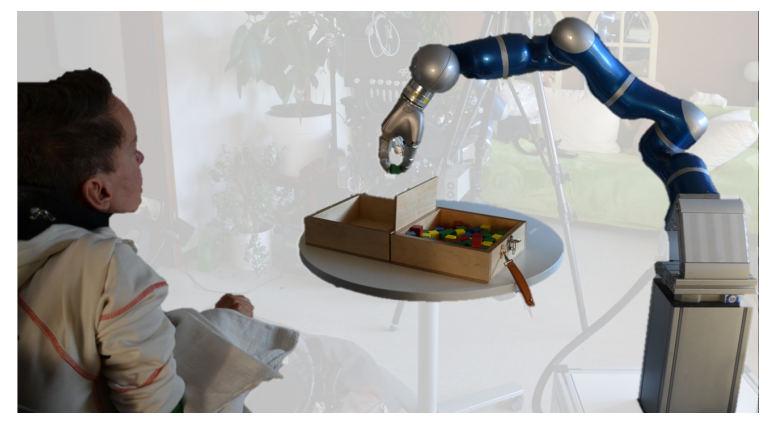

Fig. 3. S2 performing the Box and Blocks Test; sEMG-sensors are placed at the right arm which are covert with cloth to prevent the participant to feel cold.

the movement of the robot, the participants received visual feedback about their decoded commands. An additional somatosensory feedback was not included.

\section{Task description}

To evaluate the performance, two clinical assessment test were used. A modification of the Box and Blocks Test and parts of the Action Research Arm Test (ARAT).

The Box and Blocks Test is a validated performance test that assesses gross manual dexterity of the upper limb [13]. The original procedure is to move as many blocks as possible from one box to another, separated by a $10 \mathrm{~cm}$ high wall, within 1 minute. This quantitative test is widely used in the rehabilitation sector to measure the upper-limb function of patients [14]. The test is also used to measure the functional performance of myoelectric prosthesis [15], [16]. Here, we used the Box and Blocks setup to measure the performance of the sEMG-based assistive system.

The task was to move 5 of the $2.5 \mathrm{~cm}^{3}$ blocks using control mode M1, whereas the orientation of the robotic hand is adjusted to grab the Blocks from top, with a tripodal grasp (cf. Fig. 3). Success rate and completion time were used as performance metrics. No further adjustments were made to the original test. The starting position of the robot was $25 \mathrm{~cm}$ above the Box. In case the safety mode was activated, the robot was reset to the starting position. The test was performed twice in a row on all four consecutive days. Prior to each test series, a training of 5 minutes was done by the participants. On the first day an additional training of $10 \mathrm{~min}$ was done by the participants to get familiar with the control mode and the task.

The ARAT is a validated assessment test for upper limb functionality [17]. It is originally used to track the ability for activities of daily living in patients with stroke or other neurological disorders [17], [18]. The test has also been used to assess the performance of controlling a prosthetic limb using an invasive BCI [6], [7]. The original ARAT consists of 19 items grouped in grasp, grip, pinch, and gross arm movement. In this study nine items of grasp and grip subscale were considered. The standardized procedure (shown in [17]) was fulfilled except for an adaptation in the 


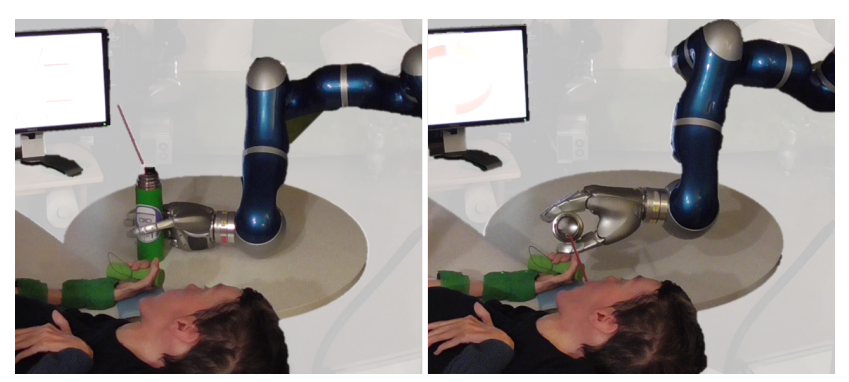

Fig. 4. S1 drinking from a bottle with a straw; sEMG-sensors are placed at the right arm; a monitor provides the information about the sub-mode of M2.

distances needed to comply with robots range of motion. The starting position of the hand was about $27 \mathrm{~cm}$ above, $46 \mathrm{~cm}$ right, and $30 \mathrm{~cm}$ behind the respective target object. The grasp subscale includes tasks of grasping a target object and place it on a box next to it. The test involves different sized cubes, a stone, and a ball. In this subscale we omitted the $10 \mathrm{~cm}$ cube due to the limited aperture of the robotic hand. The grip subscale involves diverse tasks requiring more fine manipulation. Pouring from one glass to another, displacing a tube $(1 \mathrm{~cm}$ and $2.25 \mathrm{~cm})$ from the starting peg to the target peg, and displace a washer from a tin to a bolt. The size of the washer was increased such that the DLR HIT HAND was able to grasp it $(65 \mathrm{~mm})$. The ARAT was performed using control mode M2. The initial position of the five finger hand was aligned with the $\mathrm{x}$-axis with the palm facing to the participant (c.f. Fig. 5(a)). The activation of the safety mode or the dropping of an object terminated a trial. The ARAT is an observational test, evaluating the performance with scores from 0 to 3 ( 0 : no movement; 1 : task partly done; 2 : task done, but not correctly; 3 : task done correctly. The completion of a task of more than 5 seconds is scored with 2). The completion time was used as first performance metric. Each item of the test was performed until the participant had completed the task. Prior to the first task, the participants had 15 minutes of training before the test, in order to get familiar with control mode M2. All tasks from the grasp subscale, as well as parts of the grip subscale (Tube $1 \mathrm{~cm}$ and Tube $2.25 \mathrm{~cm}$ ) were performed on the second day of experiments. The pouring and the displacement of the washer was done on the third day of experiments.

On the fourth day of experiments the participants had the possibility to serve themselves a drink with the robotic system. These task was performed with the M2 as well. The participants were outside of the robot's defined reachability at anytime. A straw located in the bottle allowed them to access the drink. Fig. 4 shows S1 picking up the bottle and drinking from it.

\section{RESULTS}

The participants could successfully complete the modified Box and Blocks Test. It took the subjects between $104 \mathrm{sec}$ to $795 \mathrm{sec}$ to move the 5 blocks from one box to the other. Within the 5 minutes of training S2 could move up to 8 blocks. Detailed times of each trial and the amount of blocks
TABLE II

Results of the BoX And Blocks Test; the Table SHOWs the AMOUNT OF BLOCKS TRANSPORTED WITHIN TRAINING (T0) AND THE COMPLETION TIMES FOR TRIAL 1 (T1) AND TRIAL2 (T2).

\begin{tabular}{cccccccccc} 
& \multicolumn{4}{c}{ Participant 1} & & \multicolumn{4}{c}{ Participant 2 } \\
\cline { 2 - 7 } \cline { 6 - 8 } day & 1 & 2 & 3 & 4 & & 1 & 2 & 3 & 4 \\
& & & & & & & & & \\
$\mathrm{t} 0[\sharp]$ & 4 & 3 & 7 & 4 & & 4 & 8 & 7 \\
$\mathrm{t} 1[\mathrm{~s}]$ & 326 & 456 & 170 & 409 & & 335 & 118 & 141 & 271 \\
$\mathrm{t} 2[\mathrm{~s}]$ & 550 & 600 & 192 & 472 & & 795 & 200 & 104 & 242
\end{tabular}

moved within the training can be seen in Table II. The time to transport a single block took between $20 \mathrm{sec}$ to $360 \mathrm{sec}$ for S1 and between $20 \mathrm{sec}$ and $246 \mathrm{sec}$ for S2.

The Action Research Arm Test assesses the full range of function of the system. Table III shows the completion time per task and participant and the needed number of trials to perform the task successfully. S1 took between $175 \mathrm{sec}$ and $430 \mathrm{sec}$ to grasp the target items of the grasp subscale from the table and position them at the top of the target box. The stone needed two trials, while all other trials were completed within the first try. In S2 all performed tasks of the grasp subscale were achieved in the first try. The average time to complete the tasks was approximately $150 \mathrm{sec}$.

All four tasks of the grip subscale were performed successfully by both participants. An exemplary task performance is depicted in Fig. 5, where S1 executes the washer-task. The completion times (listed in Table III) of this subscale reflect the complexity of the tasks. The participants typically needed two to three trials to complete a task successfully. An ARAT total score of 18 could be achieved for both participants, by assigning 2 points per successful task, without considering the time for completion.

Fig. 6 illustrates details on which of the sub-modes (translation, rotation, and grasp) were used to control the robot

TABLE III

RESULTS OF THE ARAT; SHOWN ARE COMPLETION TIMES AND THE NUMBER OF TRIALS NEEDED TO COMPLETE THE TASKS; THE REACHED ARAT-SCORE IS DEPICTED PER SUBJECT

\begin{tabular}{cccccc} 
Grasp subscale & \multicolumn{2}{c}{ Participant 1} & & \multicolumn{2}{c}{ Participant 2} \\
\cline { 2 - 3 } \cline { 5 - 6 } Item & time[sec] & trials & & time[sec] & trials \\
\hline Cube $2.5 \mathrm{~cm}$ & 430 & 1 & & 151 & 1 \\
Cube $5 \mathrm{~cm}$ & 175 & 1 & & 135 & 1 \\
Cube $7.5 \mathrm{~cm}$ & 244 & 1 & & 129 & 1 \\
Ball & 217 & 1 & & 162 & 1 \\
Stone & 262 & 2 & & 189 & 1
\end{tabular}

\begin{tabular}{cccccc} 
Grip subscale & \multicolumn{2}{c}{ Participant 1} & & \multicolumn{2}{c}{ Participant 2} \\
\cline { 2 - 3 } \cline { 5 - 6 } Task/Item & time[sec] & trials & & time[sec] & trials \\
\hline Pouring & 320 & 2 & & 320 & 3 \\
Tube $2.25 \mathrm{~cm}$ & 246 & 1 & & 196 & 2 \\
Tube $1 \mathrm{~cm}$ & 702 & 2 & & 424 & 1 \\
Washer & 467 & 3 & & 1035 & 2 \\
& & & & & \\
\hline Total score & & 18 & & & 18 \\
(of possible 30) & & & & &
\end{tabular}



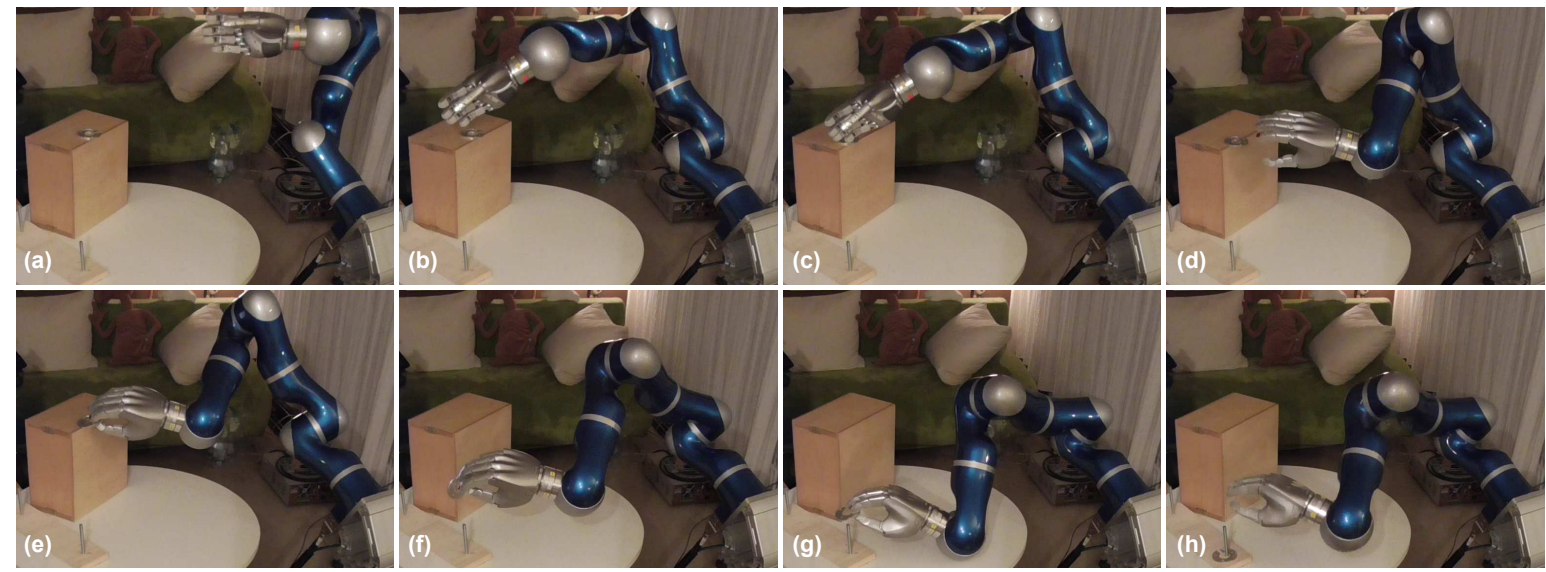

Fig. 5. Robots positions during the washer-task of the ARAT, commanded by S1; (a): the starting position of the robot; (b), (c): S1 move the washer on the edge of the box; (d): adjusted hand which allows to grip the washer; (e): the washer is picked up; (f), (g): washer is brought over target bolt; (h): task is completed. This third trial of the task took $7 \mathrm{~min} 47 \mathrm{sec}$ for completion.

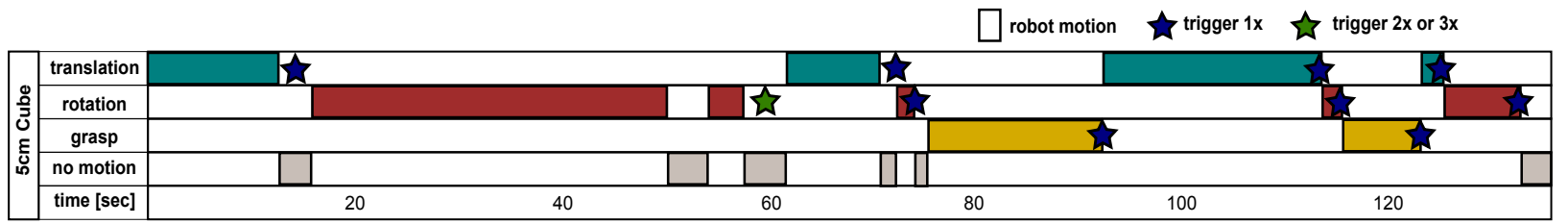

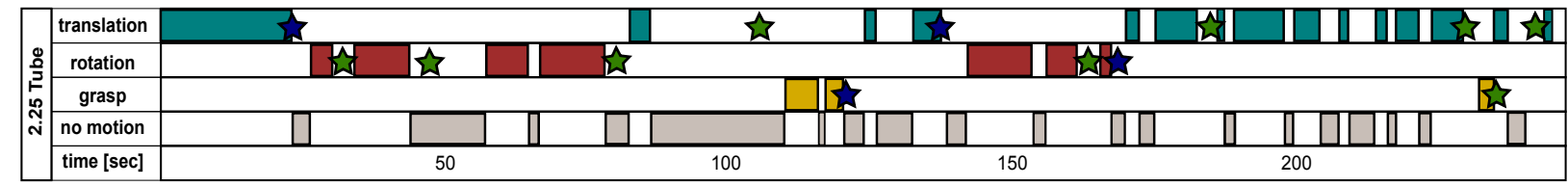

Fig. 6. The used sub-modes of M2 to control the robot during the ARAT. The mode includes translation, rotation and grasp. The decoded velocity command move the robot respectively to the selected mode. Sub-modes were changed by activating the trigger signal. The figure shows also the time, where the participant did not move the robot at all. Top: task of moving the $5 \mathrm{~cm}$ block performed by S2. Bottom: task of the $2.25 \mathrm{~cm}$ tube done by S1.

during the ARAT and when the participants paused the motion of the robot in two exemplarily chosen tasks. The upper plot shows the motion of the robot during the $5 \mathrm{~cm}$ cube task. The bottom plot shows the task of moving the $2.5 \mathrm{~cm}$ tube from the starting peg to the target peg. The displacement of the cube (upper plot) shows a continuous movement of the robot with little pauses in between. For the tube displacement significantly more pauses and shorter movements of the robot are evident. The longer pauses between individual motions suggest that the participant is planning for the optimal strategy, path and position. The rather short motions result from the desire to precisely move the robot to place the tube over the target peg.

The participants could additionally show, that they can serve them self a drink with the sEMG-based interface and the robotic arm. A bottle was picked up from the table in front of them and moved close to their mouth. A straw was used to drink from the bottle. Afterwards the bottle was placed back on the table.

\section{Discussion AND CONCLUSION}

This case study shows that paralyzed people with residual voluntary muscle activity can control a robotic hand-arm system via an sEMG-based interface to perform functional tasks. Two participants with spinal muscular atrophy could accomplish reach and grasp tasks with the provided system. Both participants used sEMG-based interfaces in the past, however, the last usage was more than two years prior at the time of this study. The overall net usage of the system is less than 5 hours before this study. The participants were able to quickly utilize the two provided control modes to fulfill clinical assessment tests for upper limb function.

The both reach and grasp tasks could show, that the participants were able to control the robot in a defined and precise way. Although the completion time was quite high, the participants were able to successfully perform all of the given tasks, some of which at the very first trial.

The modified Box and Blocks Test represented a more repetitive test. The participants were able grasp 5 blocks and move them from one box to the other with the simplified control strategy M1. The completion time for the 5 blocks in our study is quite high compared to healthy people (49years old woman: 82 block in $60 \mathrm{sec}$ [13]) or users of myoelectrical prosthesis (20 blocks in $60 \mathrm{sec}$ [16]). However, it has to be noted that performing this task with a robot arm is considerably more difficult as no tactile information 
is available to the user. Furthermore, the increased size of the hand and the comparably low velocity of the endeffector lead to longer execution times as well. Additionally, S1, who was lying in her bed during the task, had to deal with partly visual occlusion of blocks located at the bottom of the box. Still, the participants were able to control the robot precisely enough to fulfill the task.

The performed Action Research Arm Test assesses the capability to fully control the available degrees of freedom in a functional task. Both participants could show that they can handle the task with the provided system. They could reach and grasp the different items and move them to the target location. Both participant could complete all given tasks of the grasp and grip subscale. All items were performed within the first three attempts and with only a few hours of system usage. Failure of an attempt was mostly caused by exceeding the safety-force-threshold, which was difficult to perceive, as no haptic feedback was available to the participants. The completion time was less than 7 minutes within the grasp subscale and up to 17 minutes for tasks of the grip subscale. These times are relatively high in comparison to [6], where the same tasks were performed by user with an implanted BCI and a prosthetic limb. However, in that study, the BCI user had 13 weeks of training to learn control of the robot before performing the tasks.

Due to the findings shown in Fig. 6 we expect that the completion times would significantly reduce, if the participants could train regularly with the system. We assume that the pause times (no motion commanded to the robot) are used for planning how to best perform the task. This assumption has to be validated in future long term studies were the effect of daily training can be measured. In order to thoroughly investigate such long-term learning effects, experiments need to be conducted with a more consistent electrode placement, as slight changes in electrode placement may have an effect on subjects" performance. This holds also true for the Box and Blocks Test as we could not identify a training effect from the repetitions within the four experimental sessions. Comparable studies of performing the ARAT by a system using an EEG-based or eye-tracking interface could not be found in literature.

Additionally to the clinical assessment test, the participants performed tasks of their daily living. In one experimental session, the participants grasped a bottle in order to drink from a straw. The interface allowed to performing of this task without external help.

In conclusion, we could show that users of the sEMGbased interface can perform functional reach and grasp tasks. It could help them to perform simple activities of daily living without the help of a care-giver. Further long term studies have to be conducted in future to determine the effect to the performance of daily training.

\section{REFERENCES}

[1] G. Romer, H. J. Stuyt, and A. Peters, "Cost-savings and economic benefits due to the assistive robotic manipulator (arm)," in Rehabilitation Robotics, 2005. ICORR 2005. 9th International Conference on. IEEE, 2005, pp. 201-204.
[2] V. Maheu, P. S. Archambault, J. Frappier, and F. Routhier, "Evaluation of the jaco robotic arm: Clinico-economic study for powered wheelchair users with upper-extremity disabilities," in Rehabilitation Robotics (ICORR), 2011 IEEE International Conference on. IEEE, 2011, pp. 1-5.

[3] S. M. Grigorescu, T. Lüth, C. Fragkopoulos, M. Cyriacks, and A. Gräser, "A bci-controlled robotic assistant for quadriplegic people in domestic and professional life," Robotica, vol. 30, no. 3, pp. 419431, 2012.

[4] G. Onose, C. Grozea, A. Anghelescu, C. Daia, C. Sinescu, A. Ciurea, T. Spircu, A. Mirea, I. Andone, A. Spânu et al., "On the feasibility of using motor imagery eeg-based brain-computer interface in chronic tetraplegics for assistive robotic arm control: a clinical test and longterm post-trial follow-up," Spinal cord, vol. 50, no. 8, pp. 599-608, 2012.

[5] L. R. Hochberg, D. Bacher, B. Jarosiewicz, N. Y. Masse, J. D. Simeral, J. Vogel, S. Haddadin, J. Liu, S. S. Cash, P. van der Smagt et al., "Reach and grasp by people with tetraplegia using a neurally controlled robotic arm," Nature, vol. 485, no. 7398, pp. 372-375, 2012.

[6] J. L. Collinger, B. Wodlinger, J. E. Downey, W. Wang, E. C. TylerKabara, D. J. Weber, A. J. McMorland, M. Velliste, M. L. Boninger, and A. B. Schwartz, "High-performance neuroprosthetic control by an individual with tetraplegia," The Lancet, vol. 381, no. 9866, pp. 557-564, 2013.

[7] J. E. Downey, J. M. Weiss, K. Muelling, A. Venkatraman, J.-S. Valois, M. Hebert, J. A. Bagnell, A. B. Schwartz, and J. L. Collinger, "Blending of brain-machine interface and vision-guided autonomous robotics improves neuroprosthetic arm performance during grasping," Journal of neuroengineering and rehabilitation, vol. 13, no. 1, p. 28 , 2016.

[8] P. Parker, K. Englehart, and B. Hudgins, "Myoelectric signal processing for control of powered limb prostheses," Journal of electromyography and kinesiology, vol. 16, no. 6, pp. 541-548, 2006.

[9] C. Castellini and P. van der Smagt, "Surface emg in advanced hand prosthetics," Biological cybernetics, vol. 100, no. 1, pp. 35-47, 2009.

[10] J. Vogel, J. Bayer, and P. Van Der Smagt, "Continuous robot control using surface electromyography of atrophic muscles. in 2013 ieee," in RSJ International Conference on Intelligent Robots and Systems, 2013, pp. 845-850.

[11] A. Albu-Schäffer, C. Ott, and G. Hirzinger, "A unified passivitybased control framework for position, torque and impedance control of flexible joint robots," The international journal of robotics research, vol. 26, no. 1, pp. 23-39, 2007.

[12] J. Vogel, S. Haddadin, B. Jarosiewicz, J. D. Simeral, D. Bacher, L. R. Hochberg, J. P. Donoghue, and P. van der Smagt, "An assistive decision-and-control architecture for force-sensitive hand-arm systems driven by human-machine interfaces," The International Journal of Robotics Research, vol. 34, no. 6, pp. 763-780, 2015.

[13] V. Mathiowetz, G. Volland, N. Kashman, and K. Weber, "Adult norms for the box and block test of manual dexterity," American Journal of Occupational Therapy, vol. 39, no. 6, pp. 386-391, 1985.

[14] T. Platz, C. Pinkowski, F. van Wijck, I.-H. Kim, P. Di Bella, and G. Johnson, "Reliability and validity of arm function assessment with standardized guidelines for the fugl-meyer test, action research arm test and box and block test: a multicentre study," Clinical rehabilitation, vol. 19, no. 4, pp. 404-411, 2005.

[15] T. A. Kuiken, L. A. Miller, R. D. Lipschutz, B. A. Lock, K. Stubblefield, P. D. Marasco, P. Zhou, and G. A. Dumanian, "Targeted reinnervation for enhanced prosthetic arm function in a woman with a proximal amputation: a case study," The Lancet, vol. 369, no. 9559, pp. 371-380, 2007.

[16] J. S. Hebert and M. Justin Lewicke, "Case report of modified box and blocks test with motion capture to measure prosthetic function," Journal of rehabilitation research and development, vol. 49, no. 8, p. 1163, 2012.

[17] N. Yozbatiran, L. Der-Yeghiaian, and S. C. Cramer, "A standardized approach to performing the action research arm test," Neurorehabilitation and neural repair, vol. 22, no. 1, pp. 78-90, 2008.

[18] J. H. Van der Lee, V. De Groot, H. Beckerman, R. C. Wagenaar, G. J. Lankhorst, and L. M. Bouter, "The intra-and interrater reliability of the action research arm test: a practical test of upper extremity function in patients with stroke," Archives of physical medicine and rehabilitation, vol. 82 , no. 1 , pp. 14-19, 2001. 